s
533
$A_{4}$

- Secieculer Trindation 


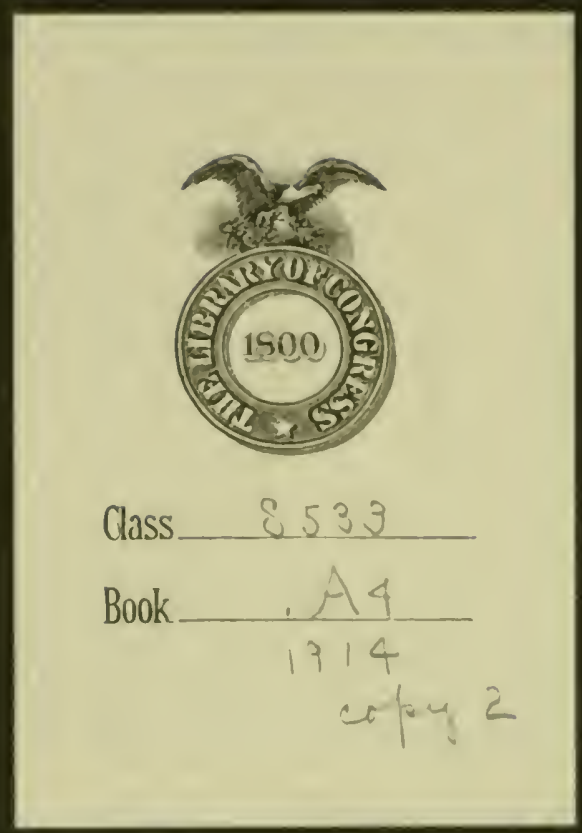




\section{ROCKEFELLER FOUNDATION}

\section{LETTER}

FROM THE

SECRETARY OF AGRICULTURE TRANSMITTING

IN RESPONSE TO A SENATE RESOLUTION OF APRIL 1, 1914, CERTAIN INFORMATION IN REGARD TO THE RELATION OF THE GENERAL EDUCATION BOARD OF THE ROCKEFELLER FOUNDATION TO THE WORK OF

THE DEPARTMENT OF AGRICULTURE

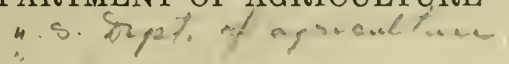

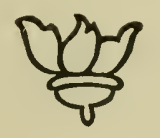

April 14, 1914.-Referred to the Committee on Printing

WASHINGTON

GOVERNMENT FRINTING OFFIOE

1914

a,pen, 2 


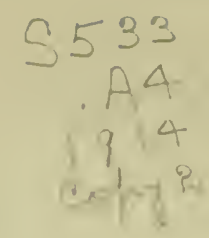

REPORTED BY MR. CHILTON.

In the Senate of the United States, July 9, 1914 .

Resolved, That the communication from the Secretary of Agriculture, in response to the resolution submitted by Mr. Kenyon and adopted by the Senate on April first, nineteen hundred and fourteen, calling for information in regard to the relation of the General Education Board of the Rockefeller Foundation to the work of the Department of Agriculture, be printed as a Senate document.

Attest:

2

JaMes M. BAKER, Secretary.

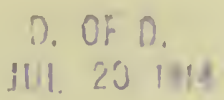




\section{ROCKEFELLER FOUNDA'TION.}

\section{The Vice President of the United States and President of the Senate. \\ April 3, 1914.}

Srr: I have the honor to transmit the information requested and directed to be furnished to the Senate by resolution adopted April 1, 1914, concerning-

1. The relation, if any, of the organization known as the General Education Board of the Rockefeller Foundation to the work of the Department of Agriculture.

2. The names and positious of all employees, if any, of the department whose salaries are paid in whole or in part with funds contributed by the Rockeleller Foundation.

3. The names and pasitions of all adminstrative officers, if any, of the department who are in any way connected with the work of the General Education Board of the Rockefeller Foundation.

4. Salaries, if any, received by them from the said Rockefeller Foundation.

The General Education Board is a corporation created by a special act of the Congress approved by President Roosevelt January 12, 1903, having as its object the promotion of education within the United States of America, without distinction of race, sex, or creed.

About the time of the incorporation of the General Education Board a serious emergency was presented in the Southwest by the ravages of the cotton-boll weevil. The Congress on January 15, 1904, made an appropriation which permitted the expenditure of $\$ 250,000$ for meeting the ravages of the weevil, including demonstration work. The demonstration work met with immediate success and attracted the attention of the General Education Board. The board expressed its desire to cooperate and offered to place at the disposal of the department additional funds for extending the work.

On April 20, 1906, after a full discussion, a memorandum of understanding was entered into between the Department of Agriculture and the General Education Board for cooperation in extending the farmers' cooperative cotton demonstration work. The agreement provided, in brief, that the work financed by the General Education Board should be distinct in territory from that carried on solely by the Department of Agriculture and that this work should be separately financed. Under the terms of the agreement the General Education Board undertook to pay the expenses directly and indirectly incurred in conducting the demonstration work in the extended territiry, and the department agreed to supervise the work and to appoint all special agents for the extended territory. It was also agreed that the agents should be under the control of the department in every respect as fully as any other agents of the department.

It was distinctly understood that the department was to be in no way responsible for the handling of the funds provided by the General Education Board, and that the department would under no consideration join with the board in payment of salaries of employees except to the extent of a nominal collaborator's salary of $\$ 1$ per annum, which was necessary and essential in order to secure the franking privilcge.

The agreement referred to has been the basis of operations since 1906. The arrangement with the General Education Board has been 
called to the atfention of the committees of (engress in the reports of the secrotary or in the reports of the Bureate of Plant Indestry, or in the public hearings hefore the committers eneh year since the work was undertaken. For the last fiseal your the bourd appopriated approximately s:50,000 for this work. 'The gronter purt of this appropriation has heren used for crencral domonstrution work in the Stalcs of Sorth Curolina, Somth Carolina, north Cicorgia, Virginia, West Vinginin, and linturky. About st5,0()0, of the amomit has

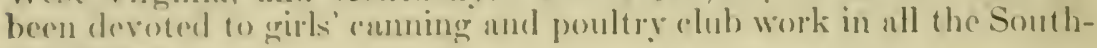
ern sitates.

The plans for the ontire work are made by the department. In comblating that work the department. us in other anses wherever pructicuble, has entered into rose relutions with the Stute collegers of ogriculture, and part of the satarios of the agents are paid from situte

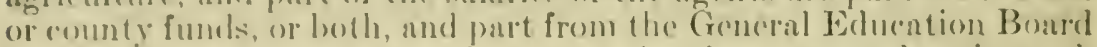
funds. In making its appointurents the departmont has in such cases aoperatod with the State rolleger.

There is appended hereto a statement giving the mames of presoms employed in farmerse (ooprentive demomstration work whose salaries ure paid in whole or in purt he the Gencinl Elusation Board. The part of the salary not so paid, with the exeoption of the collaborator's snhery of $\$ 1$ per anmmm paid by the department, jis paid, as has been inclicintol, by the sitate ar acounty, or some other local oreanization. "The statemont gives, lirst, the numes of the emplovers; seromet, the amome pre month paid hy the Gemeral Edencation Board: third, the

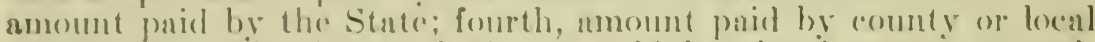
authorities: filh, the mominal sum paid hy the ibpartment: and,

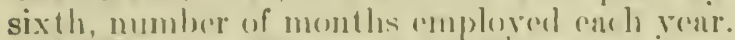

Mr. Bralford knapp is the ndministrative officer of the department who handles all the farmers' cooperntive demonstration work in the South. Is director of all the administrative work he has charge of the fores of ecollaborators who are paid in whole or in part by the Genoral Edecntion Board. He recoives no salary from the Gencral Elucation Board.

In Maret, 1913, the Congress male an appropriation for the study of marketing, and it also made an approprintion for the study of rural credits in Europe. It appeared desirable that if possible there should be an investigntion of the forms of cooperation in the Tnited states, of the problems of ruml organizntion, and espocially of the facts relating to farm eredits. These problems are intwrovern with the marketing problems and with the rumb-credit inguiry nbroad. It was suggested that it was importunt that the lepartment institute inguiries concerning these problems whilo it was investignting marketing in this comentrand ruraleredits abroal. Tho department at the time had no funds avaluble, and the (ieneral Eduention Bonrd, learning of the situntion, offered to extend its financial cooperation, and the departmont contered into an ngreement by which funds were placed at its disposal for the study of eooperntion, including rurnl credits and the general orgunization of rurnl communities for ceonomic, socinl, and edurntional purposes. The department agreed to have a director in charge of this work who should be approved by it and whose work should be directed by it, and I)r. 'Thomas Nixoi Carver, of IIarvard, was selected. It was ngrend to make him and four other necessury investigators coltaborutors, paying them n nominal salary of $\$ 1$ per 
month or per annum, as indicated below, so as to make it possible for them to use the frank:

\begin{tabular}{|c|c|c|}
\hline & $\begin{array}{l}\text { Amount } \\
\text { per month } \\
\text { paid by } \\
\text { General } \\
\text { Education } \\
\text { Board. }\end{array}$ & $\begin{array}{l}\text { Amount paid } \\
\text { by the } \\
\text { Department } \\
\text { of } \\
\text { Agriculture. }\end{array}$ \\
\hline 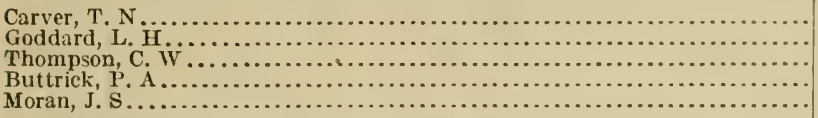 & $\begin{array}{r}\$ 625.00 \\
333.34 \\
333.34 \\
150.00 \\
125.00\end{array}$ & $\begin{array}{l}\$ 1 \text { per month. } \\
\$ 1 \text { per annum. } \\
\$ 1 \text { per month. } \\
\$ 1 \text { per month. } \\
\$ 1 \text { per annum. }\end{array}$ \\
\hline
\end{tabular}

The department also selected 12 professors of economies in agricultural colleges or universities in the country and made them collaborators with the nominal compensation of $\$ 1$ per annum, so that they might use the Government frank. Their names are:

Prof. C. B. Austin, University of Texas, Austin, Tex.

Prof. Charles Iee Raper, University of North Carolina, Chapel Hill, N. C.

Prof. L. D. H. Weld, University Farm, St. Paul, Minn.

Dean Leon S. Merrill, University of Maine, Orono, Me.

Prof. W. O. Hedrick, State College, East Lansing, Mich.

Prof. Samuel D. Gromer, University of Missouri, Columbia, Mo.

Prof. G. O. Virtue, University of Nebraska, Lincoln, Nebr.

Prof. George Thomas, Agricultural College, Logan, Utah.

Prof. A. W. Taylor, Washington State College, Pullman, Wash.

Prof. C. B. Williams, Iowa State College, Ames, Iowa.

Prof. Alexander E. Cance, Massachusetts Agricultural College, Amherst, Mass.

Prof. Morton A. Aldrich, Tulane University, New Orleans, La.

The traveling expenses of these men were paid by the general education board.

It made a similar arrangement with Dr. Lillian Johnson, of Memphis, Tenn., and Miss Ermestine Noa, of Lookout Mountain, Tenn.

The following persons are employed as clerks under Dr. Carver, their salaries being wholly paid by the general education board. They are not collaborators of the department.

Salary

per month.

Daisy F. Fridley ............................................ $\$ 85$

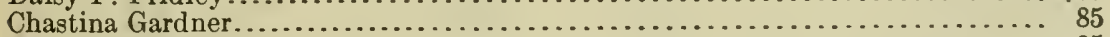

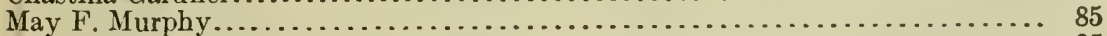

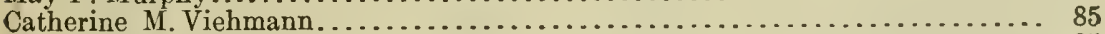

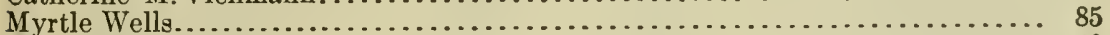

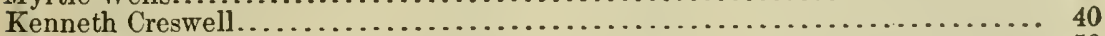

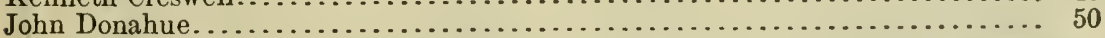

The total amount appropriated by the general education board for this purpose has been $\$ 37,166.66$.

On December 31, 1913, it was agreed that this arrangenient should terminate on June 30, 1914.

If for any reason the Congress should conclude that this farmers' demonstration work carried on in cooperation with the general education board since 1906 should be discontinued, the work in the South will have to be curtailed accordingly or an appropriation from the general revenues of $\$ 250,000$ will be required.

Respectfully,

D F. Houston, Secretary. 
Statement giting names of persons employed in farmers' cooperative demonstration work uhose sularics are paid, wholly or in part, by the General Education Board.

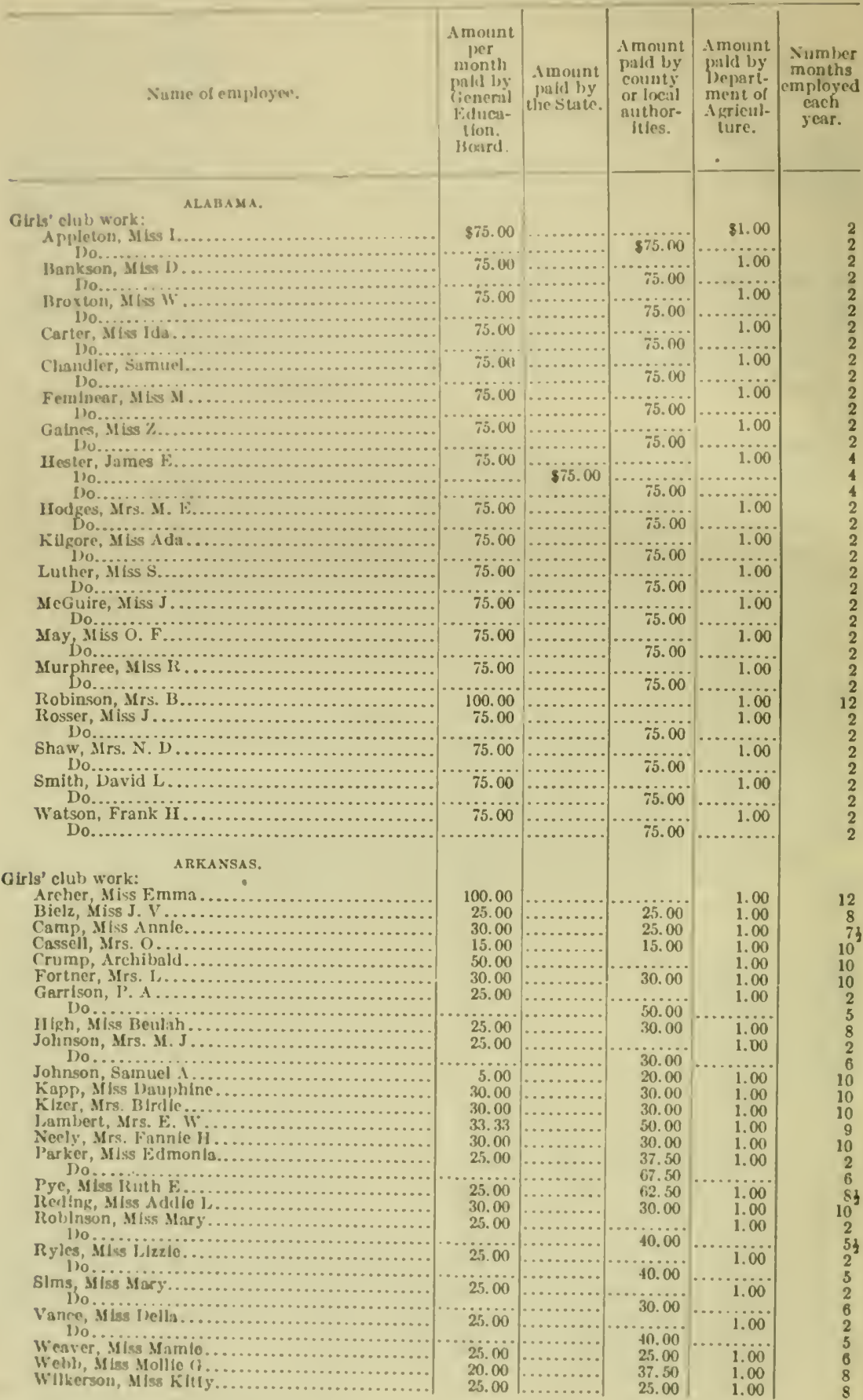


Statement giving names of persons employed in farmers' cooperative demonstration work whose salaries are paid, wholly or in part, by the General Education Board-Con.

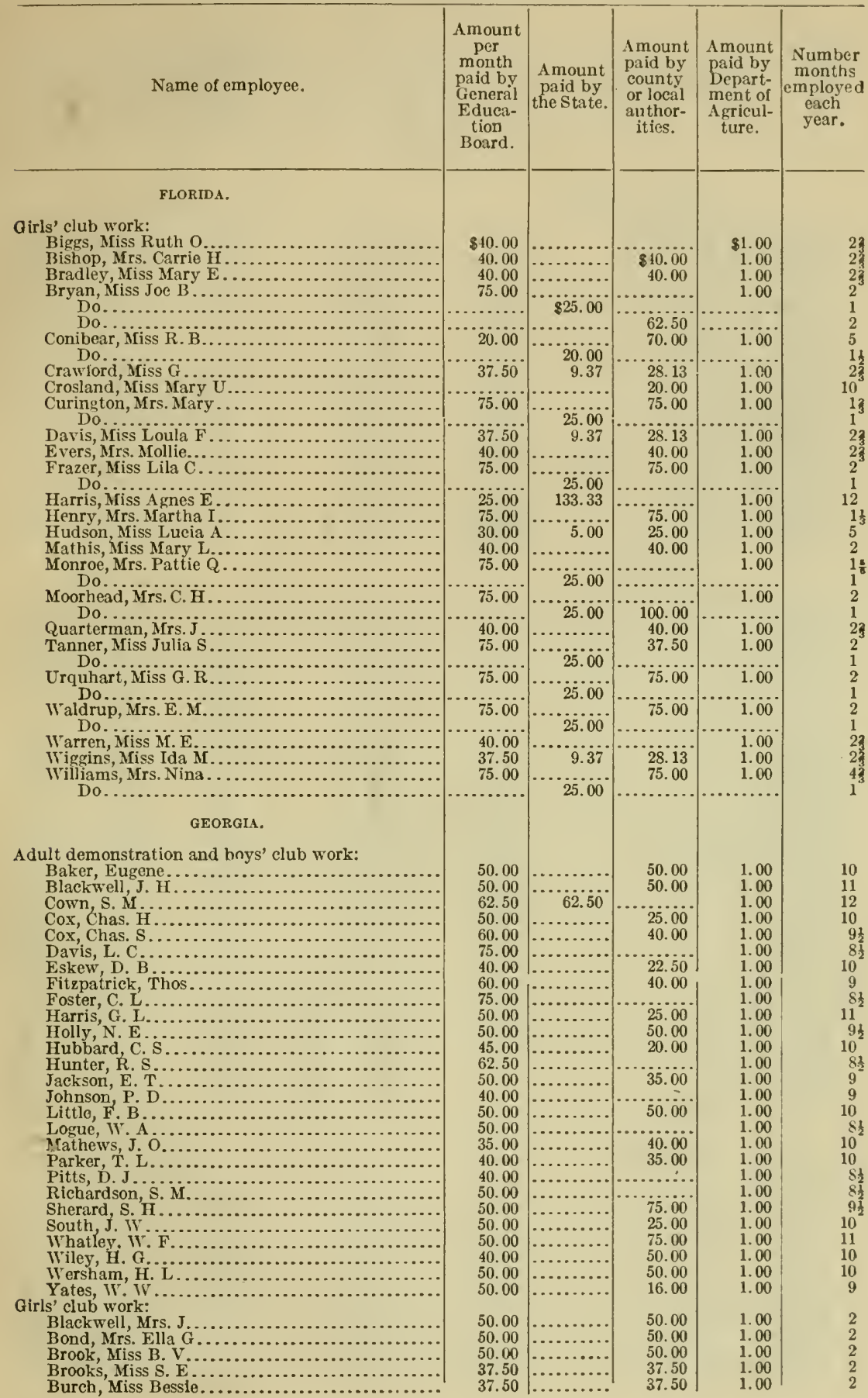


Statement giring names of persons employed in farmers' cooperative demonstration work uhose saluries are paid, wholly or in part, by the General Educalion Board-Con.

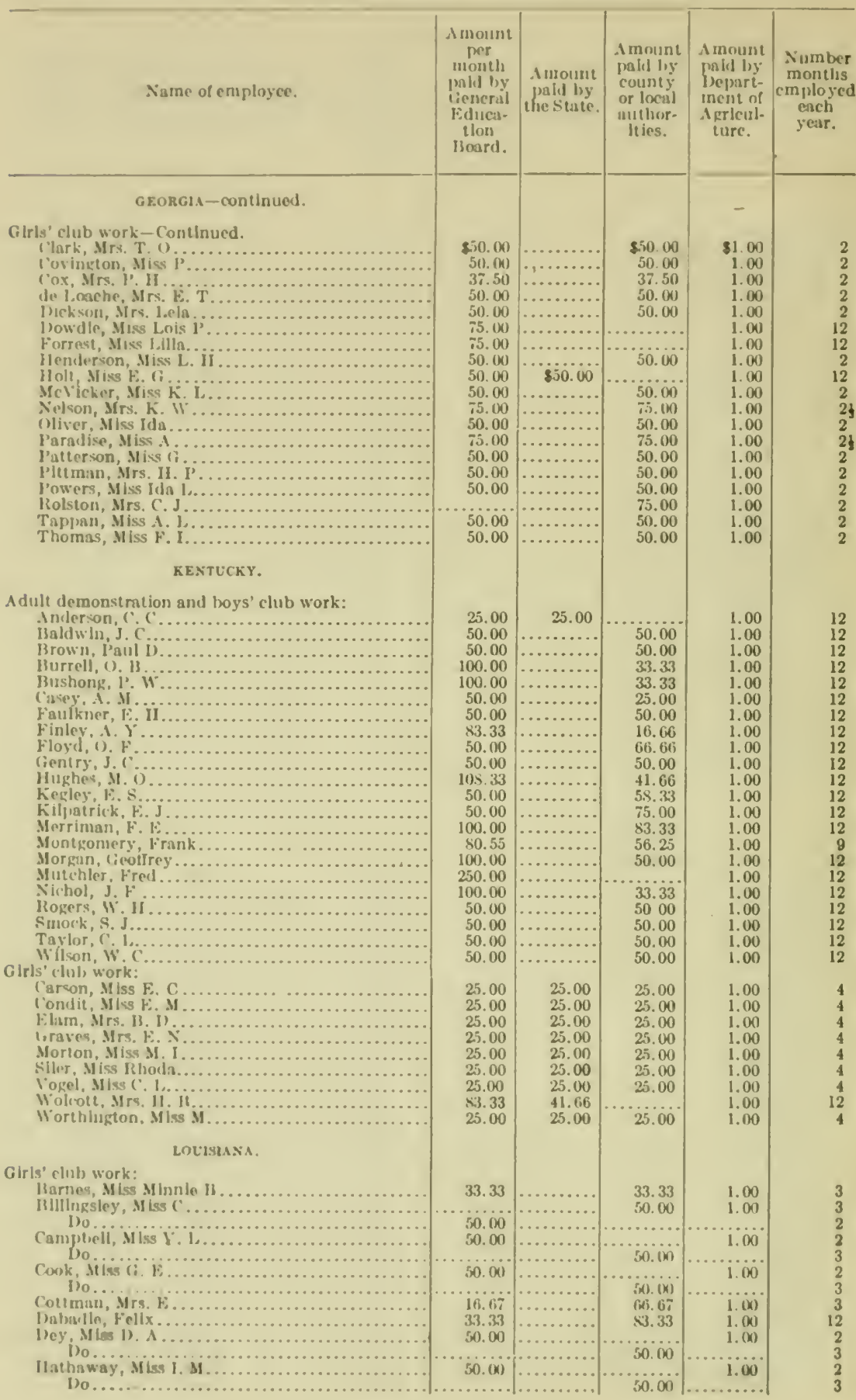


Statement giving names of persons employed in farmers' cooperative demonstration work whose salaries are paid, wholly or in part, by the General Education Board-Con.

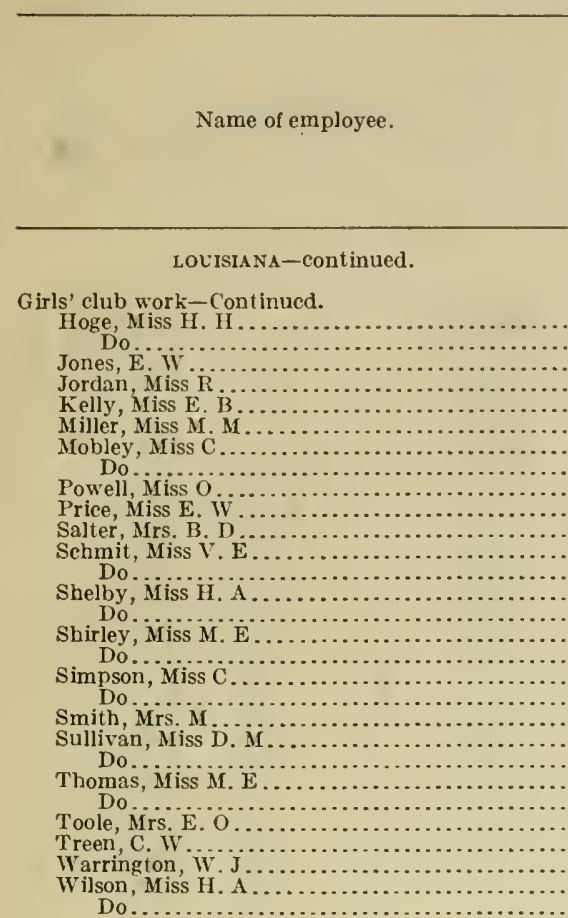

\section{MARYLAND.}

Adult demonstration and boys' club work:

Drury, John H

Graybill, Jacob $\ddot{\mathrm{L}}$.

Hodges, C. A.

Koehler, H. S.

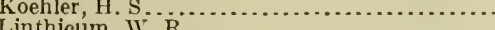

Smith, Oliver

$$
\text { Do. }
$$

Stabler, Aug

Townshend, F. C. D

Wathen, G.F., jr...

Girls' club work:

Bullock, Miss H. V

Burnite, Miss M. A

Dukes, Miss M. L...
Hamblin, Miss May

Knighton, Mrs. I. B

Linthicum, Mrs. J. M

Girls' club work:

MISSISSIPPI.

Allen, Miss Mary L

Do ..............

Berry, Mrs. J̈ennie $\mathbf{M}$.

Bolt, Miss Lena V.

Boone, Miss Annie B.

Bradley, Miss Katherine.

$$
\text { Do. }
$$

Bristow, $\mathrm{M}$ iss $\mathrm{L}$ ila $\ddot{\mathrm{M}}$

Brown, Miss Mary A.

Dockery, Miss Sarah Do.

Edwards, Mrs. Lela.

.

Fugler Miss Mittie

Graves, Mrs. Cora $\mathrm{E}$.

.

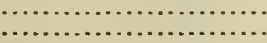

....

...........................

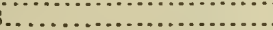

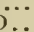

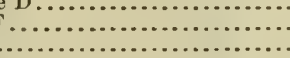

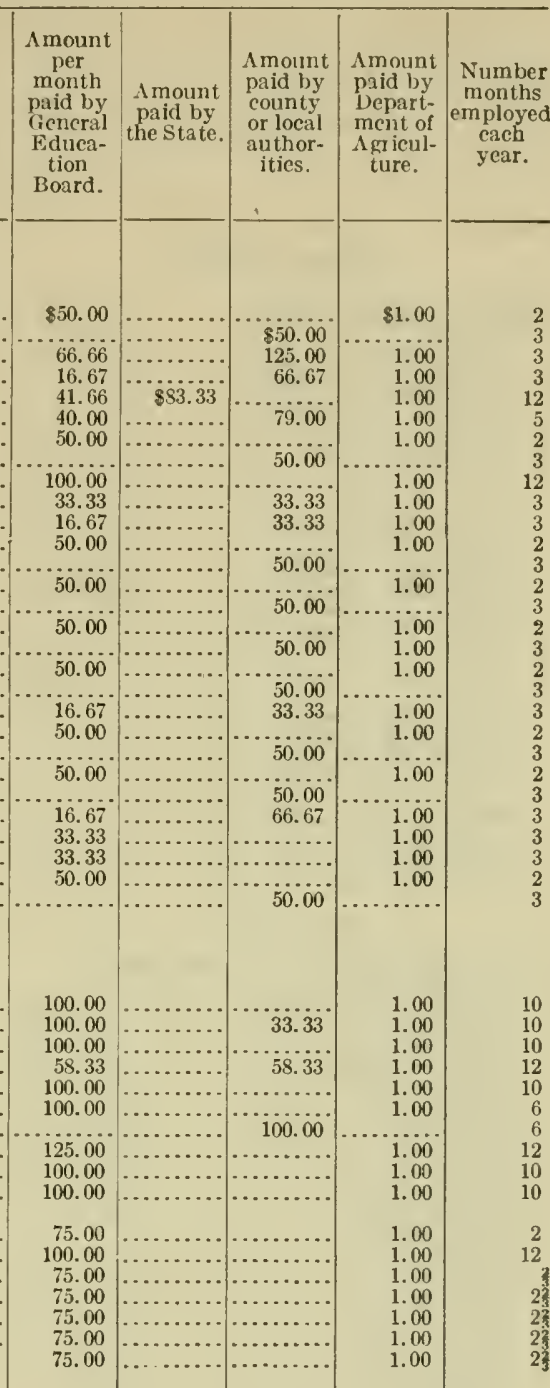

25.00

50.00

25.00

25.00

50.00

2.3 .00

25. 00

50. 00

25.00

33. 33

50.00

25.00 
Statement giving names of persons employed in furmers' conperative demonstration work whose salurics are paid, "holly or in part, by the General Education Board-Con.

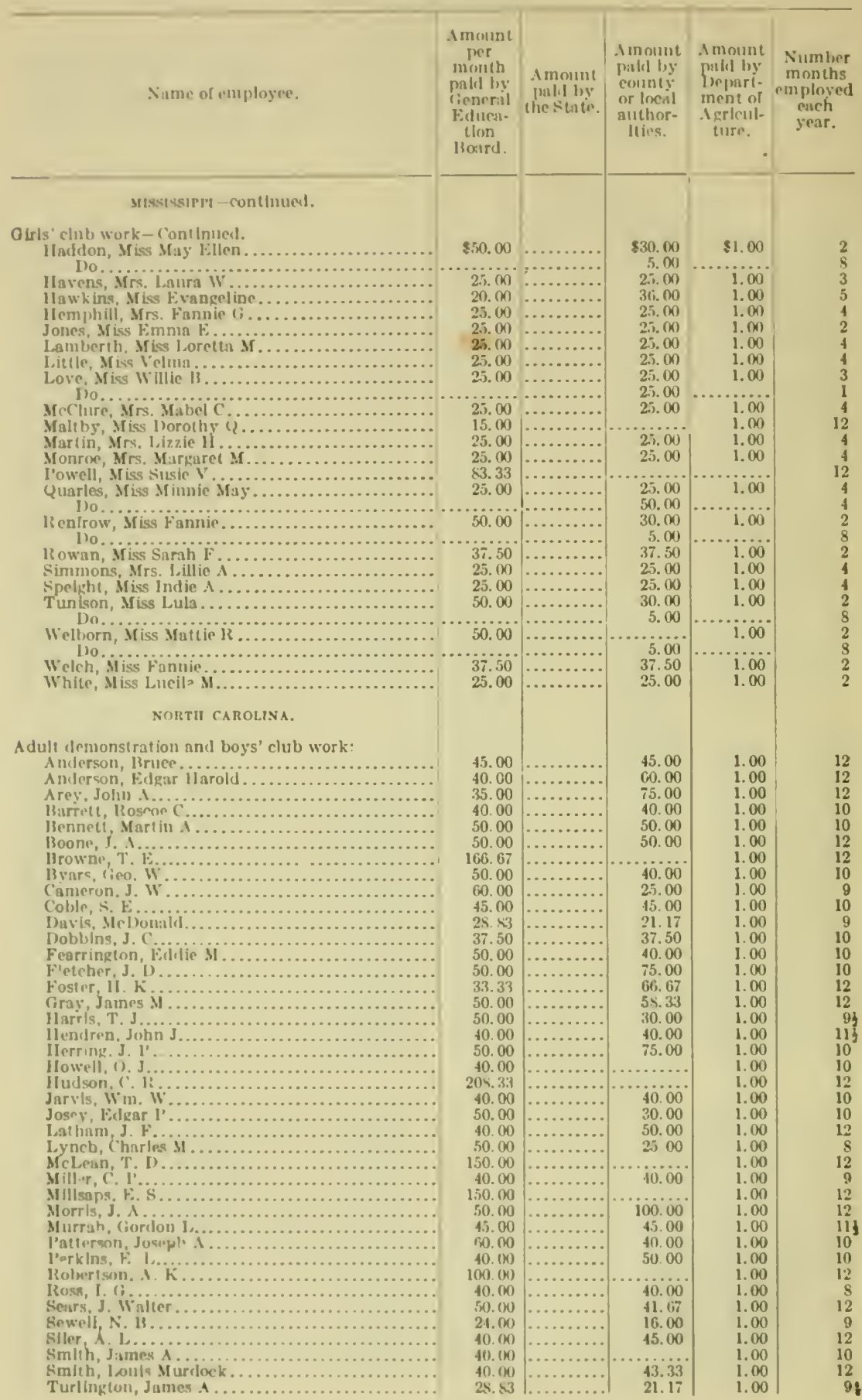


Statement giving names of persons employed in farmers' cooperative demonstration work whose salaries are paid, wholly or in part, by the General Education Board-Con.

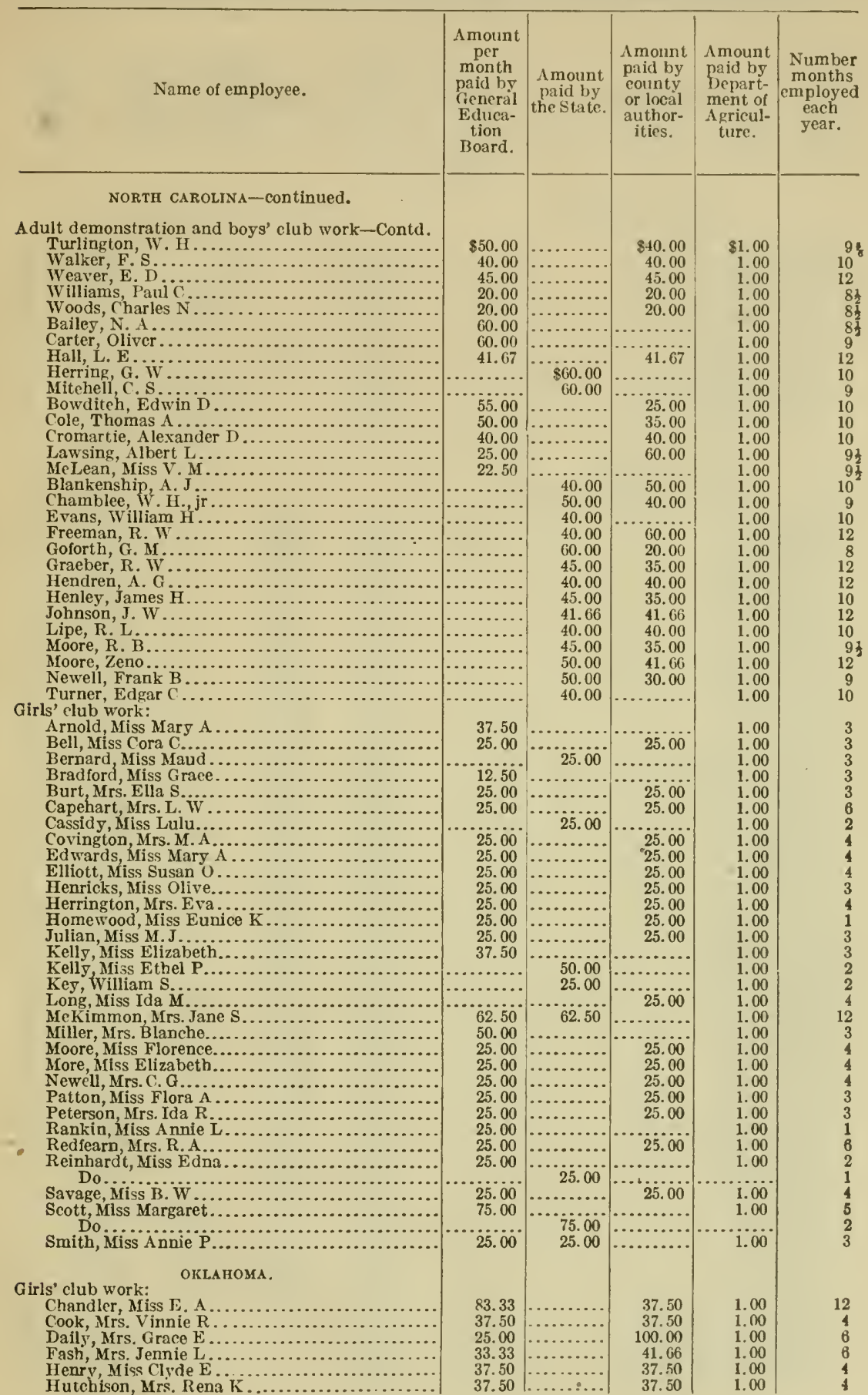


Statement giving names of fersons employed in farmers' cooperative demonstration work uhose salaries are paid, wholly or in part, by the General Education Board-Con.

Ninme of employec.

OKLABOSIA-continued.

Oirls' club work-Comtinued.

Jackison, Mrs. Lilla 'T........

MeValib, Mrs. (i. I.

Monre, Mis Verule E.

Niblack, Mrs. Mary $V^{\prime}$

Nipjer, Mrs. $\mathrm{k} . \mathrm{s}$

Ninn, Mrs. Beisla $J$

Veters, Mrs. Innie.

Kosse, Mrs. Jattie I

Sarment, IIrs. Ilenrietta.

Smill, lllss Katherine.

Tatum, Mrs. Lydia 'T'.

Whitney, JIrs. 1. B.

\section{SOUTII C.AROINA.}

Adult demonstration and boys' rlub work:

Altman, L. B...

Arrents 5.11 .

Bakir, 1.. T, .

Barton, iv, ii.

Blair, John $K$.

l3owen, T. А...

Carbery, James I

Carmell, F. IV

Chitty, I1, I3

Claity, J. F.....

Cunningham, R. I

Elliote, It. IR.

Fipps, T. (i)

Eizell, J. F.

Finklor, J, ?

Framplon, 1 , Мi

llanna, liporde 1

llayes, J. M.

Jipat, Ashton.

llearses, i. J .

IIolland, .11. $\mathrm{C}$

IItison, I3. M

Lemmnn, R, II

l.ever, 15. T. J

Long, 11.16

1.ote, 1, X.

Mc.l Ihany, !1. L,

Mcriuney, Frunk

McFuddin, C. A.

Mckeown, A....

Mclsurin, rolln

MeMamu, (. I.

Mills, T. II.

Mulirow, J. I

Ninpler, J. M

I'rlee, B. Ii

Rejil. s. F

Reulumbutm Sinmuet. .

lilley, J. A

Rothrenck,j. is

Sianders, 1. I

Shmly, J. W.

stewurt, W.

Tarrane, 1). 1.

Tiller, W..j.

Wnll, M. IV

Wette, I, I), I

Wirlsti, s, N

Willuins, J. F

I3ates, Jas. A. (colored)

13) anton, J, F. (colored)

Jenkins, F.. I). (colorm)

Jones, ( II. (cotored).

Wiley, J. ( . (colored).

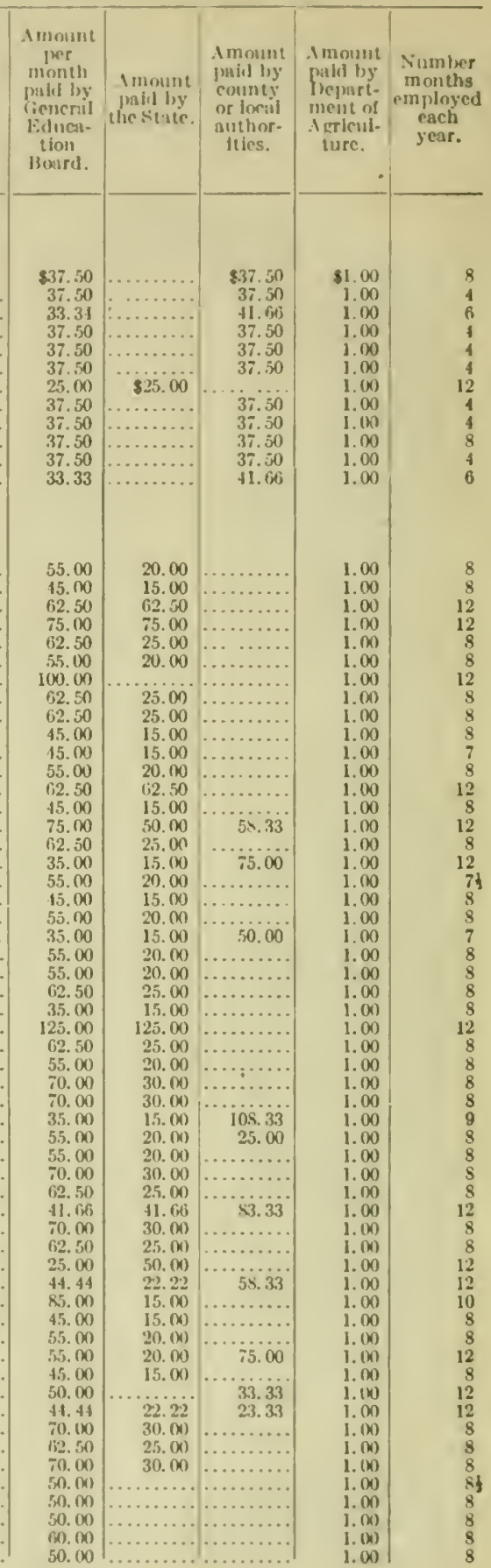


Statement giving names of persons employed in farmers' cooperative demonstration work whose salaries are paid, wholly or in part, by the General Education Board-Con.

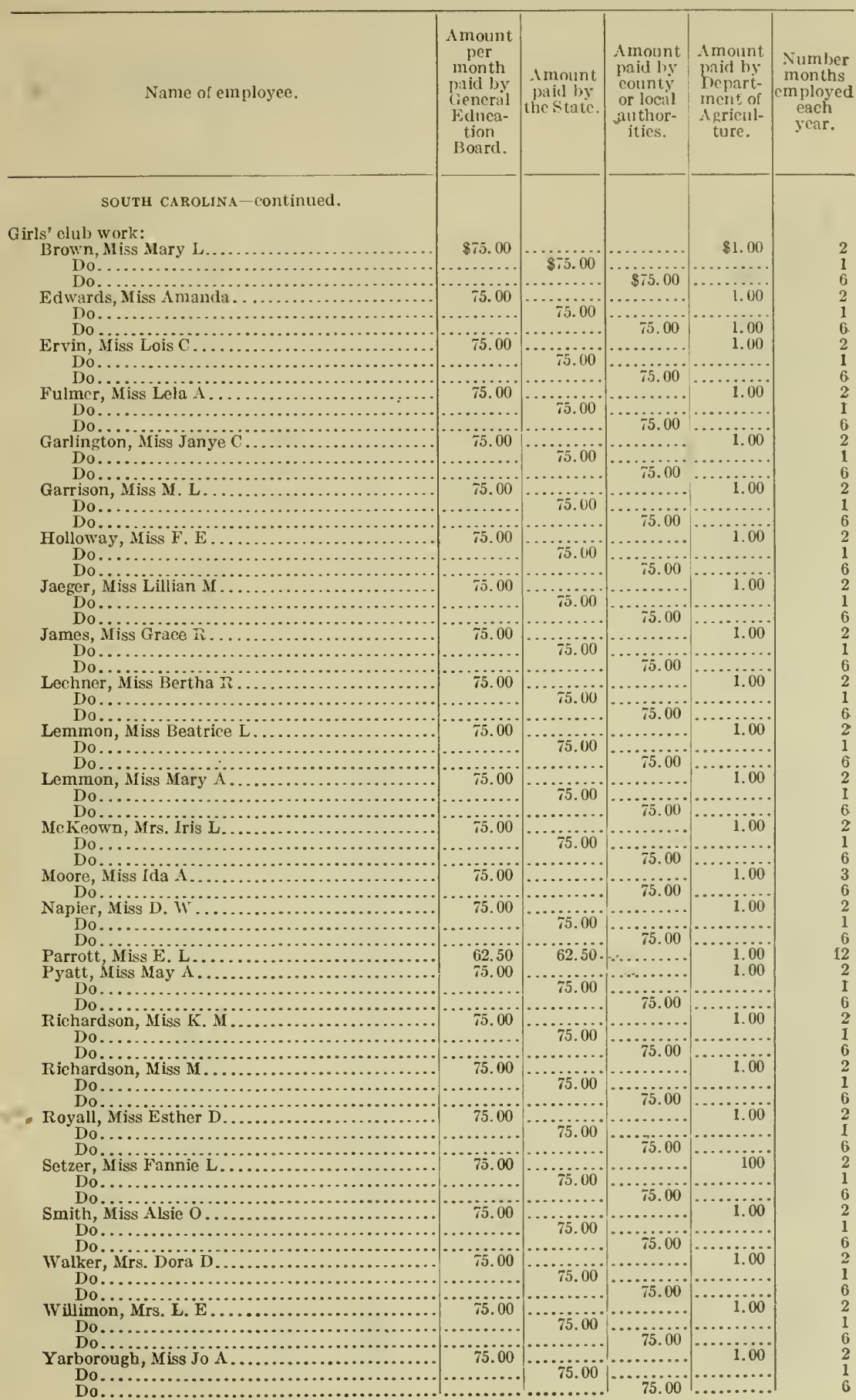


Statement giving names of persons employed in farmers' cooperative demonstration work whose sularies are paid, wholly or in part, by the General Education Board-C'on.

\begin{tabular}{|c|c|c|c|c|c|}
\hline Name of employee. & $\begin{array}{l}\text { Amount } \\
\text { jer } \\
\text { month } \\
\text { puld by } \\
\text { firmeril } \\
\text { liduen. } \\
\text { tlon } \\
\text { Bonrd. }\end{array}$ & $\begin{array}{l}\text { A mount } \\
\text { pald by } \\
\text { tho State. }\end{array}$ & $\begin{array}{l}\text { Imount } \\
\text { pald by } \\
\text { county } \\
\text { or locil } \\
\text { author- } \\
\text { lties. }\end{array}$ & $\begin{array}{l}\text { Amoint } \\
\text { fuld by } \\
\text { Dejurt- } \\
\text { ment of } \\
\text { Aericil- } \\
\text { ture. }\end{array}$ & $\begin{array}{c}\text { Nimber } \\
\text { months } \\
\text { einployed } \\
\text { each } \\
\text { year. }\end{array}$ \\
\hline \multicolumn{6}{|l|}{ Qirls' club work: TENNESSEE. } \\
\hline 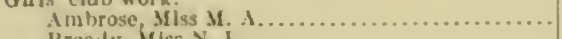 & $\$ 25.00$ & & & $\$ 1.00$ & 4 \\
\hline 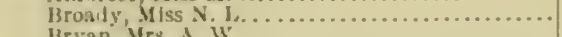 & 12.50 & $\$ 12.50$ & $\$ 25.00$ & 1.00 & 8 \\
\hline 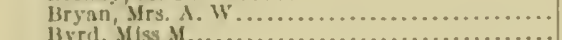 & 12.50 & 25.00 & 50.00 & 1.00 & 8 \\
\hline 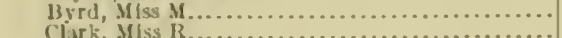 & 50.00 & $\cdots . . . \cdots$ & $\cdots \cdots \cdots$ & 1.00 & 7 \\
\hline 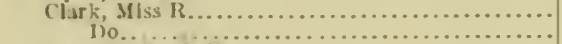 & 25.00 & 25.00 & 25.00 & 1.00 & $\begin{array}{l}4 \\
8\end{array}$ \\
\hline 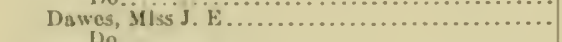 & 25.00 & $\cdots \cdots$ & $\ldots \ldots \ldots$ & 1.00 & 4 \\
\hline Intlon, & 12.50 & & $\begin{array}{l}25.00 \\
12.50\end{array}$ & $\cdots \cdots, \ddot{0}^{\prime}$ & 2 \\
\hline 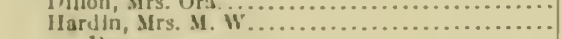 & 30.00 & & 12.50 & $\begin{array}{l}1.00 \\
1.00\end{array}$ & $\begin{array}{l}4 \\
5\end{array}$ \\
\hline & ........ & $\ldots \ldots \ldots$ & 25.00 & .......... & 8 \\
\hline 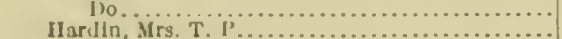 & $\cdots \cdots \cdots$ & 25.00 & $\cdots \cdots$ & & 4 \\
\hline 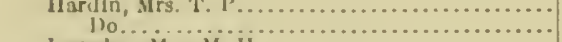 & $\begin{array}{c}25.00 \\
\ldots \ldots\end{array}$ & $\cdots 25.00$ & $\cdots \cdots, 00$ & 1.00 & $\begin{array}{l}6 \\
8\end{array}$ \\
\hline 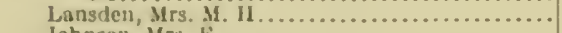 & 50.00 & & & 1.00 & 4 \\
\hline 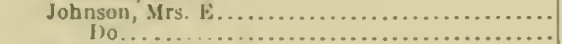 & 25.00 & 25.00 & & 1.00 & $\begin{array}{l}4 \\
8\end{array}$ \\
\hline 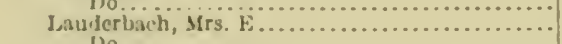 & $\ddot{25} .00$ & - $\cdots$ & $\begin{array}{c}20.00 \\
\ldots \ldots\end{array}$ & $\ddot{1.00}$ & 8 \\
\hline 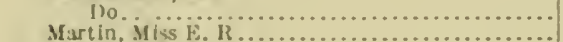 & & & 100.00 & & 12 \\
\hline 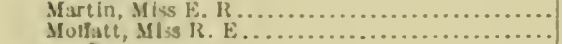 & $\begin{array}{r}8.33 \\
25.00\end{array}$ & & $\ldots \ldots$ & 1.00 & 12 \\
\hline & 25.00 & $\cdots \cdots$ & 70.000 & 1.00 & $\begin{array}{r}4 \\
10\end{array}$ \\
\hline 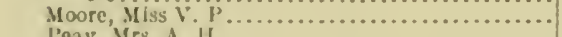 & 62.50 & ......... & 62.50 & 1.00 & $\begin{array}{l}10 \\
12\end{array}$ \\
\hline 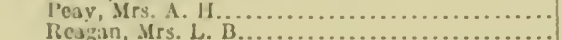 & 25.00 & $\ldots \ldots$ & 25.00 & 1.00 & 4 \\
\hline 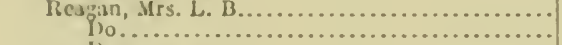 & $\begin{array}{c}50.00 \\
\ldots \ldots\end{array}$ & $\ldots \ldots$ & $\cdots$ & 1.00 & 2 \\
\hline 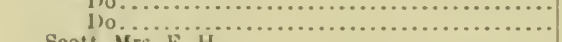 & $\cdots \cdots \cdots$ & 50.00 & 50.00 & . & $\begin{array}{l}4 \\
2\end{array}$ \\
\hline 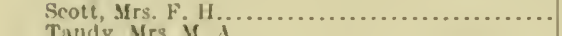 & 25.00 & .......... & & & 4 \\
\hline 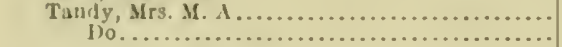 & $\cdots \cdot$ & 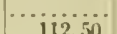 & 112.50 & 1.00 & 8 \\
\hline & $\cdots$ & & $\ldots$ & ........ & 4 \\
\hline \multicolumn{6}{|l|}{ Oirls' cluh work: TEX.AS. } \\
\hline 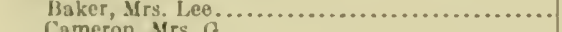 & 75.00 & & & 1.00 & 2 \\
\hline 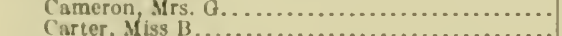 & 45. 00 & $\cdots$ & 30.00 & 1.00 & 31 \\
\hline 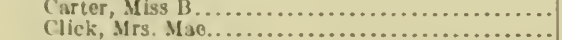 & $\begin{array}{r}100.00 \\
37.50\end{array}$ & $\cdots \cdots$ & & 1.00 & 12 \\
\hline 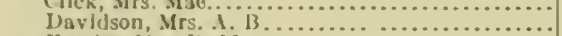 & $\begin{array}{l}37.50 \\
37.50\end{array}$ & 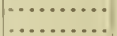 & $\begin{array}{l}37.50 \\
37.50\end{array}$ & $\begin{array}{l}1.00 \\
1.00\end{array}$ & 4 \\
\hline Frazjer, Mrs. 1) M. . . . . & 37.50 & $\cdots \cdots \cdots$ & $\begin{array}{l}37.50 \\
37.50\end{array}$ & $\begin{array}{l}1.00 \\
1.00\end{array}$ & 4 \\
\hline Marden, Mrs. Nice.......................... & 37.50 & $\ldots \ldots \ldots$ & 37.50 & $\begin{array}{l}1.00 \\
1.00\end{array}$ & 4 \\
\hline 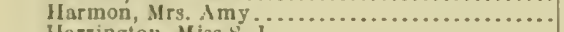 & 37.50 & $\ldots \ldots \ldots$ & 37.50 & $\begin{array}{l}1.00 \\
1.00\end{array}$ & 4 \\
\hline 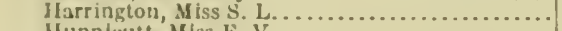 & 37.50 & .......... & 37.50 & $\begin{array}{l}1.00 \\
1.00\end{array}$ & 4 \\
\hline 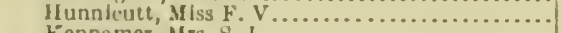 & 37.50 & $\ldots \ldots \ldots$ & 37.50 & $\begin{array}{l}1.00 \\
1.00\end{array}$ & $\begin{array}{l}4 \\
4\end{array}$ \\
\hline 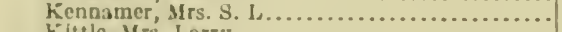 & 37.50 & $\ldots \ldots \ldots$ & 37.50 & 1.00 & 4 \\
\hline 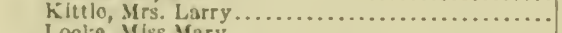 & 37.50 & $\ldots \ldots \ldots$ & 37.50 & 1.00 & $\begin{array}{l}4 \\
4\end{array}$ \\
\hline Locke, Miss Mary . . . . . . . . . . . . . . . . . . . . & 37.50 & $\ldots \ldots \ldots$ & 37.50 & 1.00 & 4 \\
\hline 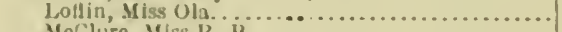 & 37.50 & .......... & 37.50 & 1.00 & 4 \\
\hline 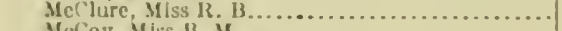 & 37.50 & $\ldots \ldots \ldots$ & 37.50 & 1.00 & 4 \\
\hline 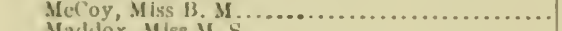 & 75.00 & $\ldots \ldots \ldots$. & & 1.00 & $\begin{array}{l}4 \\
2\end{array}$ \\
\hline 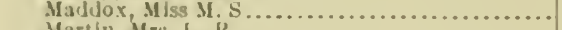 & 37.50 & $\ldots \ldots \ldots$ & 37.50 & 1.00 & 4 \\
\hline 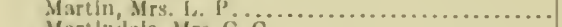 & 37.50 & $\ldots \ldots \ldots$ & 37.50 & 1.00 & 4 \\
\hline Mart Indale, Mrs. C. C....................... & 37.50 & .......... & 37.50 & 1.00 & 4 \\
\hline 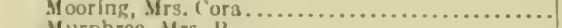 & 50.00 & .......... & 25.00 & 1.00 & 3 \\
\hline 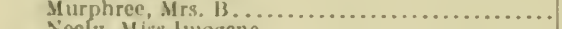 & 37.50 & $\ldots \ldots \ldots$ & 37.50 & 1.00 & 4 \\
\hline 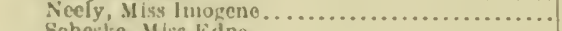 & 37.50 & $\ldots \ldots \ldots$ & 37.50 & 1.00 & 4 \\
\hline 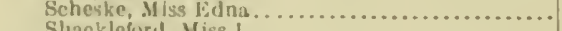 & 37.50 & $\ldots \ldots \ldots$ & 37.50 & 1.00 & 4 \\
\hline 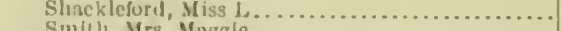 & 37.50 & $\ldots \ldots \ldots \ldots$ & 37.50 & 1.00 & 4 \\
\hline Smilti, Mrs. Magglo........................ & 37.50 & $\ldots \ldots \ldots$ & 37.50 & 1.00 & 4 \\
\hline 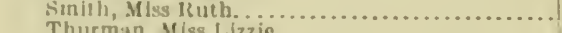 & 37.50 & ( ......... & 37.50 & 1.00 & 4 \\
\hline Thurman, Mlss Lizzie...................... & 37.50 & $\ldots \ldots \ldots$ & 37.50 & 1.00 & 4 \\
\hline 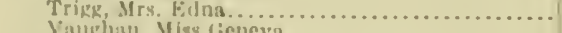 & 75.00 & ......... & & 1.00 & 2 \\
\hline 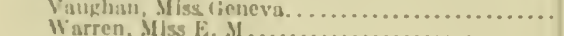 & 37.50 & .......... & & 1. 00 & 4 \\
\hline Warren, Mliss F. .l ............................ & 37.50 & ( $\ldots \ldots \ldots$ & $3 \vec{i} .50$ & 1.00 & 4 \\
\hline VIILGINIA. & & & & & \\
\hline \multicolumn{6}{|l|}{ Adult deisonstritlun and boys' club work: } \\
\hline 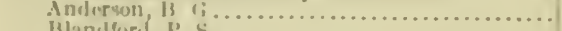 & 25.00 & 25.00 & $\$ 3.33$ & 1.00 & 12 \\
\hline 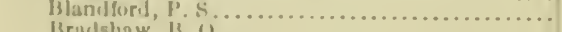 & 163.67 & 113.67 & $83.6+1$ & 1.00 & 12 \\
\hline 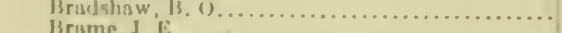 & 25. $(9)$ & 25.00 & 50.00 & 1.00 & 12 \\
\hline$\ldots \ldots \ldots \ldots \ldots \ldots \ldots \ldots \ldots \ldots$ & 21.25 & 21.25 & +2.50 & 1. (x) & 12 \\
\hline encen & 22.50 & 22.50 & 45.00 & 1.00 & 10 \\
\hline
\end{tabular}


Statement giving names of persons employed in farmers' cooperative demonstration work whose salaries are paid, wholly or in part, by the General Education Board-Con.

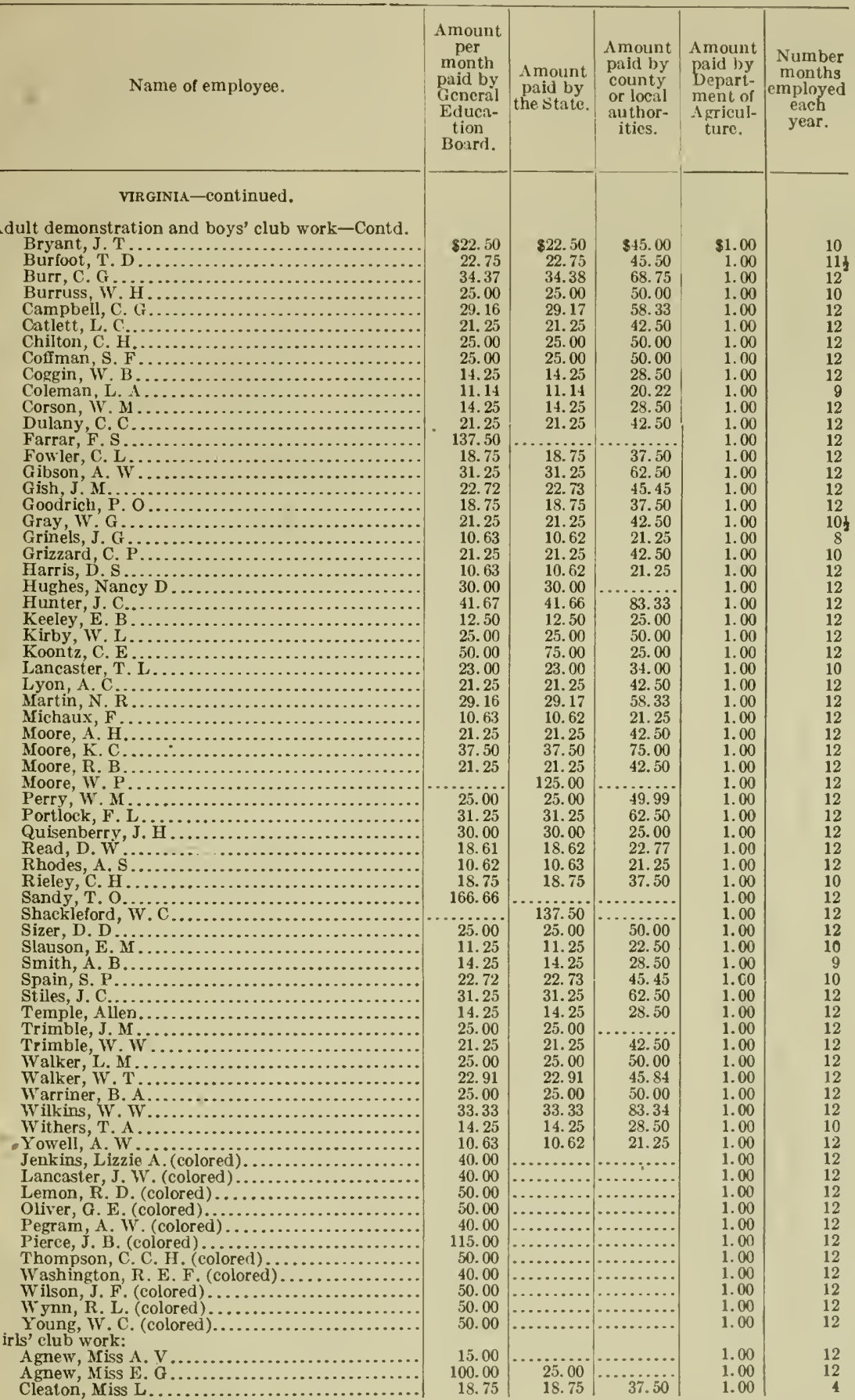


Statement giring names of persons cmployed in furmors' cooperative demenstration uork whose salaries are paid, "holly or in part, by the Cineral Eduention Board-Con.

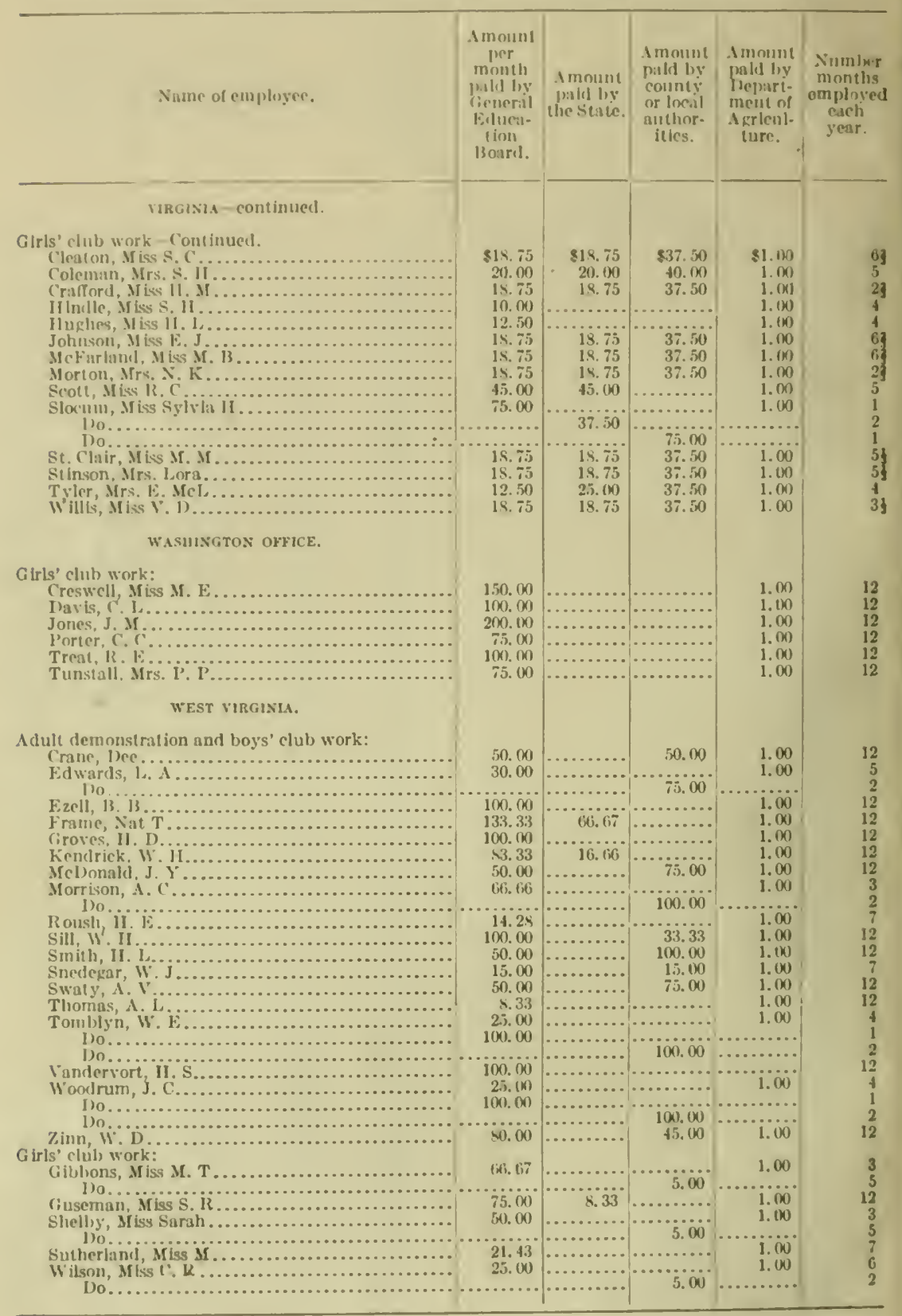


Caslord Bros

Makers

Syracuse, N. I

PAT. JAN. 21, 1908 
